\title{
Schlesische BibliotheKen \\ Zeichen DeR INTELLeKTUELlen Vielfalt einer zentralen \\ BiLdungsRegion in Europa
}

\section{Detlef Haberland}

Die Einrichtung von Bibliotheken wurde in der Frühen Neuzeit durch mehrere Faktoren begünstigt. Zum einen war es die Renaissance, während der in größerem Maße als bisher nichtklerikale Schichten an der Bildung teilhatten. Der Aufschwung der Wissenschaften und die Abkehr von der Scholastik bewirkten eine Steigerung der Textproduktion. Und schließlich waren es die Erfindung des Buchdrucks und die Reformation mit allen ihren bekannten Folgen, die zu einer gesteigerten Verbreitung von Texten beitrugen. ${ }^{1}$ Die Notwendigkeit, mehr als nur eine Bibel und einen Kalender im Hause zu haben, lag für die Gelehrten auf der Hand. Die Ausweitung von Gelehrsamkeit im Gefolge der von Melanchthon ausgerufenen „Bildungs-Offensive” und der Hinwendung zur Antike schuf zusätzliche Notwendigkeiten: Schul- und Lehrbücher mussten geschaffen werden, überhaupt waren zahlreiche biblische Bücher und antike Werke neu aufzulegen als Material für Lehrer und Schüler. Die Gelehrten benötigten zuverlässige Textausgaben. ${ }^{2}$ Was während des Mittelalters und durchaus noch bis ins 16. Jahrhundert hinein gebräuchlich war, nämlich die handschriftliche Kopie von Texten, wich zunehmend dem Buchdruck mit mehreren Auflagen.

Soweit eine grobe Skizze der Situation des Buchdrucks als Voraussetzung der Bildung von größeren Bibliotheken. Schlesien nahm, was die Entwicklung der Bildung betraf, im Heiligen Römischen

Zu diesem Thema siehe den Tagungsband von Andrea Seidler, István Monok (Hg.): Reformation und Bücher. Zentren der Ideen, Zentren der Buchproduktion. Wiesbaden, 2020, Harrassowitz (Wolfenbütteler Abhandlungen zur Geschichte des Buchwesens, 51.) (im druck)

2 In diesem Punkt ist es in erster Linie die Offizin von Aldus Manutius d.Ä. (1449-1515) in Venedig, die den Buchmarkt, was die Klassikerausgaben betrifft, revolutionierte. 
Reich eine gewisse Sonderstellung ein. Für den hier beschriebenen Zusammenhang wichtig ist der Zeitraum 1335 bis 1526, als Schlesien unter böhmische Herrschaft kam. ${ }^{3}$

Wegen des Aussterbens der direkten Linie der Přemysliden 1306 verlangsamte sich die Schlesienpolitik der Krone Böhmen, die 1311 an Johann von Luxemburg gelangt war. Erst am 6. Januar 1327 übertrug der Breslauer Herzog Heinrich VI. sein Gebiet an Böhmen, die restlichen schlesischen Herzogtümer folgten bis 1368. 1335 und 1348 verzichtete der polnische König Kasimir III. der Große auf Ansprüche der königlichen Linie der Piasten auf das alte Herzogtum Schlesien als Gegenleistung für den Verzicht der böhmischen Könige aus dem deutschen Haus Luxemburg auf die polnische Krone. Am 7. April 1348 inkorporierte schließlich König Karl IV. die schlesischen Teilherzogtümer, mit Ausnahme des Herzogtums Schweidnitz-Jauer, in die Länder der Böhmischen Krone. Damit wurde Schlesien mittelbar ein Teil des Heiligen Römischen Reichs. Herzöge und Fürstbischof hatten jedoch keinen Sitz und Stimme auf den Reichstagen und waren somit nur Böhmen untertan. Im 14. und frühen 15. Jahrhundert konnte sich Schlesien in jeder Hinsicht ungestört weiterentwickeln.

Die gegen Katholiken und Deutsche gerichteten Hussitenkriege trafen Schlesien als katholisch und deutsch geprägtes Nebenland Böhmens jedoch besonders hart: Menschen- und Siedlungsverluste, wirtschaftlicher Niedergang waren die Folge. Die Situation verbesserte sich erst 1469, als König Matthias Corvinus Mähren, Schlesien und die Lausitz eroberte und im Frieden von Olmütz 1479 gegen Böhmen in seinem Besitz bestätigt wurde. Er setzte einen allgemeinen Landfrieden durch und reorganisierte und zentralisierte die Landesverwaltung. Nach Corvinus' Tod 1490 wurde Schlesien wieder ein Lehen des Königs von Böhmen, Ladislaus II. aus der Dynastie der Jagiellonen.

Die recht gut erforschte Geschichte Schlesiens wird hier dargestellt nach Ludwig Petry (Hg.): Geschichte Schlesiens. Stuttgart, 1. Bd.: Von der Urzeit bis zum Jahre 1526. 6., unveränd. Aufl. 2000; 2. Bd.: Die Habsburger Zeit 15261740. 3., unveränd. Aufl. 2000. 
Diese Entwicklung der Politik hatte für das Bildungswesen eine grundlegende Konsequenz. Zwar bestätigte König Wladislaw von Böhmen in einem Stiftungsbrief von 1505 die Gründung einer Universität in Breslau, die durch die Jesuiten maßgeblich unterstützt wurde. ${ }^{4} 1638$ nahmen sie den Betrieb eines Gymnasiums in Breslau auf. Im Gegensatz zu Oberschlesien waren Niederschlesien und Breslau jedoch weitgehend protestantisch, so dass es naturgemäß zu erheblichen Widerständen gegen eine jesuitische Universität kam, die ihre Eröffnung verhinderten. Erst 1702 wurde sie als habsburgisch-katholische Landesuniversität mit einem Privileg Kaiser Leopolds I. gegründet.

Das bedeutete für die Anlage von Bibliotheken, dass von früh an eine zentrale akademische Stätte fehlte, in der sich Buchproduktion und -wissen hätte konzentrieren können. ${ }^{5}$ Gegenbeispiele sind etwa Prag (1348 gegründet), Krakau (1364), Heidelberg (1386). Leipzig (1409), stets schon früh mit wichtigen Bibliotheken. Auch die Universität in Tyrnau/Trnava in Oberungarn war eine Gründung der Jesuiten (1635), die älteste in Ungarn, die unter Maria Theresia 1777 nach Buda verlegt wurde. ${ }^{6}$

$\mathrm{Da}$ es also weder eine Universität noch eine entsprechende zentrale Bibliothek in Schlesien gab, blieb den Gelehrten, gleich welcher Konfession oder Fachrichtung, nichts anderes übrig, als eigene Bibliotheken zusammenzustellen. Darüber hinaus gab es natürlich weiterhin Klosterund Kirchenbibliotheken, Adelsbibliotheken wie auch solche von

Siehe hierzu Norbert Conrads (Hg.): Die tolerierte Universität. 300 Jahre Universität Breslau 1702 bis 2002. Katalogbuch zur Ausstellung. Wiesbaden 2004, v.a. S. 17-83 mit einem historischen Abriss.

Siehe dazu Klaus Garber: Schlesiens Bildungslandschaft zwischen Barock und Aufklärung im Kontext des Späthumanismus. In: Marek Ha£ub, Anna MańkoMatrsiak (Hg.): Ślaska Republika Uczonych. Schlesische Gelehrtenrepublik. Slezská Vědecká Obec. Vol. 1. Wrocław 2004, S. 288-301. Natürlich gab es verschiedene Druckereien in Schlesien. Der Buchdruck begann in Breslau 1475, in Oels 1527 (eine hebräische Druckerei), in Liegnitz 1528, in Neisse nach einem vereinzelten Druck 1541 regulär 1555, in Hundsfeld (eine hebräische Druckerei) 1543, in Görlitz 1545. Siehe dazu Detlef Haberland: Schlesien. In: Severin Corsten, Stephan Füssel, Günther Pflug (Hg.): Lexikon des gesamten Buchwesens. LGB ${ }^{2}$. Bd. 6. Stuttgart 2003, S. 549-555. Heute heißt sie Eötvös Loránd Tudományegyetem. 
Lateinschulen und Gymnasien. ${ }^{7}$ Diese vier Bibliotheksformen sind durch die bildungsgeschichtliche Position Schlesiens begründet und haben sich in den folgenden Jahrhunderten immer stärker ausdifferenziert.

Zwei historische Einschnitte haben diese Entwicklung nachhaltig verändert: Die Säkularisation 1810, die Johann Gottlieb Gustav Büsching für die Klosterbibliotheken in Schlesien durchführte (auf Ochsenkarren wurden unzählige Bücher aus Kirchen- und Klosterbibliotheken nach Breslau transportiert und Dubletten aussortiert), und das Ende des Zweiten Weltkriegs 1945, als Schlesien unter polnische Verwaltung fiel und die Bibliothekslandschaft neu geordnet werden musste. Da es im Rahmen dieses Beitrags kaum möglich ist, alle genannten Bibliothekstypen ausführlich zu beschreiben, sollen im Folgenden jeweils besondere Beispiele herausgegriffen und exemplarisch charakterisiert werden. ${ }^{8}$

Hier seien die vier wichtigen Gymnasialbibliotheken in Breslau genannt, die sich aus Kirchenbibliotheken entwickelten: Die von St. Elisabeth, St. Maria Magdalena, St. Bernhardin und St. Mathias. Sie behielten ihre Bedeutung auch nach der Gründung der Universität in Breslau bei.

8 Der diesem Beitrag zugrundeliegende Vortrag sowie die vorliegende Ausarbeitung basieren, was die schlesischen Bibliotheken betrifft, vor allem auf folgendem Werk: Detlef HABERLAND (in Verbindung mit Weronika KarLAK und Bernhard Kwока): Kommentierte Bibliographie zum Buch- und Bibliothekswesen in Schlesien bis 1800. München 2010 (Schriften des Bundesinstituts für Kultur und Geschichte der Deutschen im östlichen Europa, Bd. 39). - Die dort aufgeführten 1.652 Arbeiten zu allen Bereichen der Druck-, Buch-, Papier-, Einband-, Buchhandels-, Lese- und Bibliotheksgeschichte umfassen den Zeitraum vom 15. Jahrhundert bis ca. 1800 und repräsentieren die Forschungen vor allem in Polen, Deutschland und Tschechien. Vor allem die zahlreichen kleineren Quellen sind mit Signaturnachweisen aus der Biblioteka Uniwersitecka w Wrocławiu (Universitätsbibliothek Breslau) versehen. In den Kommentaren wird versucht, so genau wie möglich, Inhalte, Daten und Namen wiederzugeben, so dass ein Eindruck von der jeweiligen Forschungsleistung, aber auch von der Sache an sich (Druckort, Bibliothek, Papiermühle etc.) entsteht. Personen-, Orts- und Sachregister erschließen den gesamten Bestand. Es ist aufschlussreich, dass vom Herbst 2010, als diese Bibliographie erschien, bis zur Abfassung dieses Beitrags kaum noch neue Werke zu diesem Thema erschienen. Hier dürfte sich ein Wandel der Forschungsinteressen andeuten, obwohl noch zahlreiche Probleme gerade der Buchgeschichte offen sind. - Alle Zahlen in diesem Beitrag sind den Kommentaren der Bibliographie entnommen; sie sind durch die Struktur des Werkes leicht aufzufinden und werden daher im Folgenden nicht im Einzelnen nachgewiesen. 
Zunächst zu den Klosterbibliotheken. In Breslau hatten das Domkapitel trennen, das Dominikanerkloster und das Jesuitenkolleg ihren Sitz. Nach einem Katalog von 1786 hatte die Bibliothek des Domkapitels einen Umfang von 6.300 Titeln (ohne Handschriften) $(605)^{9}$ mit vielen Werken des 15. und 16. Jahrhunderts. Schon 1776 wurden rund 960 Bücher versteigert, und in diesem frühen Katalog heißt es, dass die Bibliothek durch den schwedischen Einmarsch 1632 schwer in Mitleidenschaft gezogen worden war (604). Die Bibliothek der Dominikaner umfasste zur Zeit der Säkularisation bereits ca. 10.000 Titel (738) mit einer Vielzahl von Werken dominikanischer Autoren. Die Jesuitenbibliothek ist wohl nicht durch einen vollständigen Katalog dokumentiert. (739) Es gibt nur Fragmente von Katalogen. $\mathrm{Zu}$ nennen sind wenigstens noch die Klosterbibliotheken von St. Christoph, St. Dorothea, St. Vinzenz und der Corpus-Christi-Kirche mit kleineren Beständen. St. Bernhardin hatte hingegen eine bedeutend umfangreichere Bibliothek.

Auch außerhalb von Breslau war in den Klöstern teilweise ein beachtlicher Buchbestand vorhanden. Das Jesuitenkloster in Brieg etwa besaß um 18001.336 Bücher, hauptsächlich Kirchenväter, kaum jedoch humanistische oder naturwissenschaftlichen Werke. Ein beträchtlicher Zuwachs kam 1690 durch zahlreiche evangelische Bücher und Bibeln zustande. Die Bibliothek wurde 1801 in die Universitätsbibliothek eingegliedert (1003).

Natürlich gab es auch Bibliotheken in kleineren Klöstern: In Czarnowanz und Glatz, in Glogau (Kollegiatskirche, Dom, Jesuiten) mit z.T. beachtlichen Beständen. Nicht selten waren die Kanoniker selbst bibliophile Gelehrte, was sich an ihren eigenen Bibliotheken zeigt. In Goldberg gab es eine Kettenbibliothek der Stadtpfarrkirche mit einer Reihe von Rara. Das Zisterzienserklostern in Grüssau besaß nach dem Katalog von 1812 ca. 2.600 Titel, zumeist aus dem 17. und 18. Jahrhundert (1206). Auch sie wurde von den Schweden geplündert und im Rahmen der Säkularisation von Büsching zerstreut, d.h. auf andere 
Bibliotheken verteilt (1208). Heinrichau als Mutterkloster Grüssaus besaß eine beträchtliche Bibliothek, die nach Carl Heinrich Rother ein „bedeutender Markstein und qualitativer Orientierungspunkt für andere Klosterbibliotheken der Region"war (1212). Klosterbibliotheken gab es auch in Hirschberg, in Jakobskirche, Leubus, Liegnitz (hier bemerkenswert für die Pfarrkirche St. Peter und Paul ist die Gelegenheitsschriften-Sammlung mit ca. 20.000 Titeln, darunter ca. 3.400 Leichenpredigten) (1295). Neisse als der Sitz der katholischen Bischöfe besaß mit den Bibliotheken von St. Jakob, des Franziskanerund Jesuitenklosters bedeutende bibliophile Schatzhäuser. Die erstere ist heute im Domarchiv in Breslau vorhanden (1385). Schließlich seien wenigstens noch die kleineren Kirchenbibliotheken von Oels, Oppeln, Rauden, Sagan, Schweidnitz, Teschen, Trebnitz, Troppau und Wohlau genannt. Diese besaßen zumeist jeweils sehr viel weniger Bücher und bedienten vor allem die theologischen Bedürfnisse der Pfarrer; teilweise ragen hier aber auch besondere Kostbarkeiten heraus. So besaß die Saganer Klosterbibliothek der Augustiner Chorherren insgesamt ca. 7.000 Bücher, davon waren etwa 250 Inkunabeln (1486).

Von den Schulbibliotheken (zu denen hier auch die der Ritterakademie in Liegnitz gerechnet wird) sind vor allem die in Breslau hervorzuheben. Es sind die bereits genannten Bibliotheken des Elisabeth-Gymnasiums, des Maria-Magdalena-Gymnasiums [Авв. 1], des Matthias-Gymnasiums und des Bernhardiner Gymnasiums. Die Bibliothek des Elisabeth-Gymnasiums (621-650) ist eine der Hauptquellen, aus denen die spätere Breslauer Stadtbibliothek entstand. Diese Gymnasialbibliothek ging aus der Kirchenbibliothek von St Elisabeth hervor, eine der protestantischen Hauptkirchen Breslaus; ihre Bestände reichen bis in Mittelalter zurück und wurden dann qualitativ entscheidend durch die Bibliothek des Patriziers und Sammlers Thomas Rehdiger (1540-1576) (860-870)10 erweitert. Sein

10 Siehe dazu Detlef Haberland: Thomas Rehdiger - Humanist, Sammler und Begründet der Breslauer Stadtbibliothek. In: Marek Haєub, Anna MańkoMatysiak (Hg.): Śląka Republika Uczonych. Schlesische Gelehrtenrepublik. Slezská Vědecká Obec. Vol. 4. Wrocław 2010, S. 73-112. 


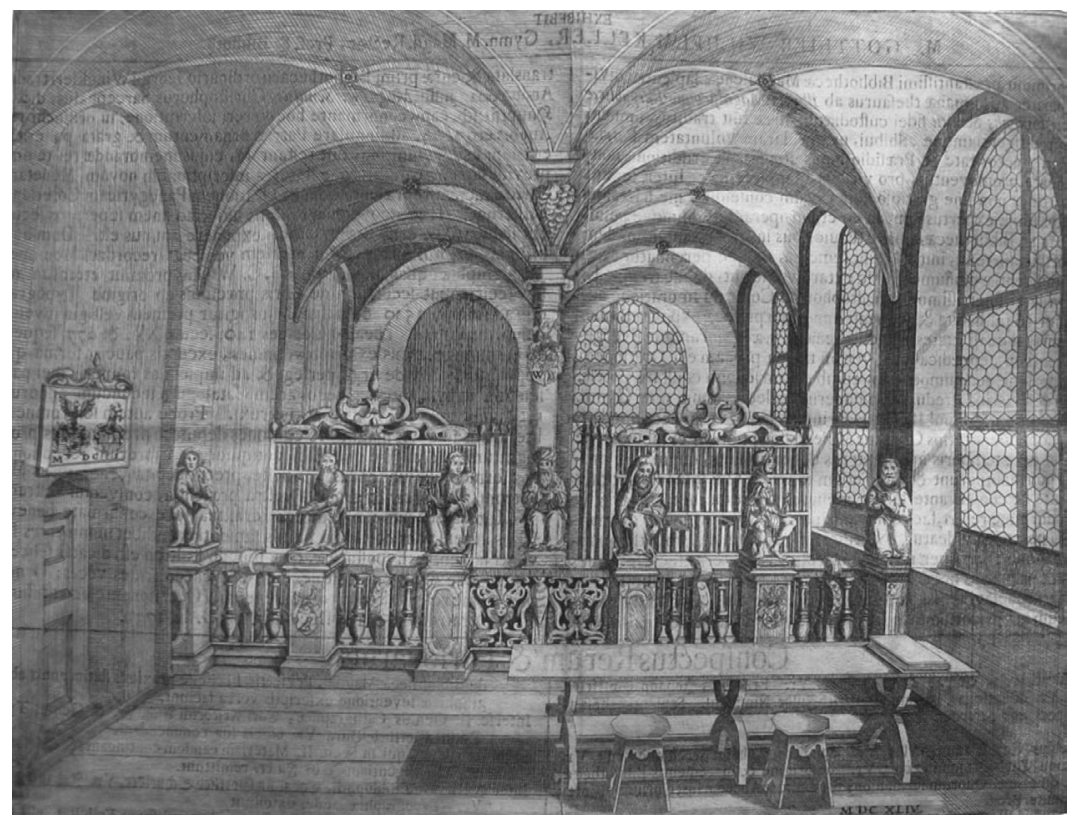

Abb 1. Das Innere der Maria-Magdalena-Bibliothek in Breslau. Kupferstich aus Wilhelm Gottlieb Keller: Memorabilium Bibliothecae Mariae Magdalenae partem primam ante solemnem praemiorum [...] distributionem in Vratislaviensium Gymnasio

Magdaleneo d. XVII. Dec. M DCC XXXIX. per selectos studiorum cultores [...].

Vermächtnis umfasste bereits ca. 6.000 Bücher und 300 Handschriften [Авв. 2]. Eine Reihe von weiteren wichtigen Legaten bereicherten die Bibliothek zusätzlich: Es sind schlesische Adlige und Bürger wie Johanna Susanna von Ohl und Adlerskron, Johann Gottlieb Uber, Gottlob Kranz, Albrecht von Sebisch, Heinrich von Reichel, Johann Caspar Arletius u.a. - diese hochgebildeten und jeweils auf bestimmte Gebiete fokussierten Gelehrten bereicherten die Schulbibliothek mit ihren gelehrten Spezialbibliotheken. Die Bibliothek ist durch verschiedene ältere Kataloge und Repertorien noch aus dem 18. Jahrhundert teilweise, aber noch nicht vollständig erschlossen. Sie umfasste 1863 ca. 42.500 Titel aus praktisch allen Disziplinen (748-766). Die Entwicklung der 


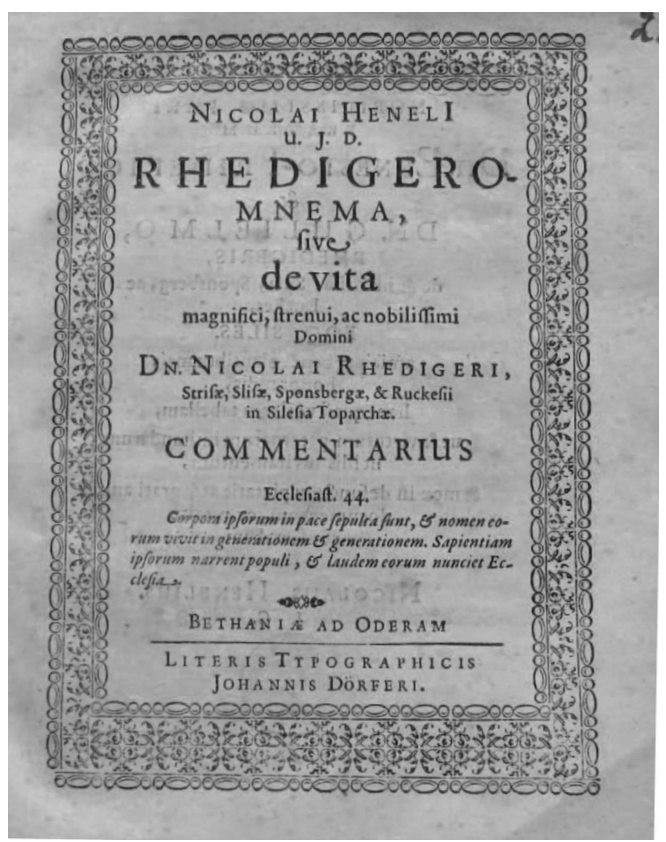

Abb. 2. Würdigungsschrift des Geschlechtes derer von Rhediger: Nicolai Henelii: Rhedigeromnema sive de vita [...] Nicolai Rhedigeri [...] commentarius. Beuthen an der Oder [1616]. Biblioteka Uniwersytecka w Wrocławiu, Sign. BUWr 534275.

Stadtbibliothek ist durch verschiedene Kataloge und auch durch Versteigerungskataloge von Dubletten gut erschlossen.

Auch die Schulbibliothek des Maria-Magdalena-Gymnasiums ist eine der zentralen und bedeutenden Schulbibliotheken Breslaus gewesen; sie verdankt ihre Entstehung wohl der Bibliothek des schlesischen Reformators Johannes Hess (1490-1547), der sie der Kirchengemeinde St. Maria Magdalena stiftete. Auch für sie liegt eine Reihe von Katalogen vor. Durch verschiedene Legate Breslauer und schlesischer Bürger ist sie ebenfalls ungemein bereichert worden, z.B. durch das des lutherischen Theologen und Gymnasialprofessors an St. Elisabeth, Johann David Raschke (1676-1760), mit 1.455 Büchern (686) wie auch durch das des ehemaligen Rektors des Maria-Magdalena-Gymnasiums, Johann Caspar 
Friedrich Manso (1759-1826), der der Bibliothek 4.490 Bücher aus seinem Besitz hinterließ (687); des Letzteren Wissens- und Sammelschwerpunkte waren Geographie, Pädagogik und Philologie.

Für beide Bibliotheken gilt jedoch, dass eine Erschließungsarbeit erst noch geleistet werden müsste. Das Handbuch deutscher historischer Buchbestände in Europa ${ }^{\text {ll }}$ gibt zwar einen Überblick über die Fachgebiete, kann aber naturgemäß keine qualitativen Aussagen zu einzelnen Bestandsgruppen machen. Für beide Bibliotheken kann man aber sagen, dass sie Bücher aus allen Wissensgebieten enthalten und bei weitem nicht nur theologische Werke. Philologie, Jura, Geschichte, antike Klassiker sind reichhaltig vorhanden, dazu Inkunabeln aus allen Bereichen in großer Zahl.

Schließlich soll noch die Bibliothek des Bernhardiner Gymnasiums genannt werden, die ebenfalls aus einer Kirchenbibliothek erwuchs. Sie war wohl die größte dieser Kirchen- und Schulbibliotheken mit ca. 68.000 Titeln im Jahre 1865.

Es ist leicht nachvollziehbar, welche geballte Wissenskonzentration allein in diesen drei Bibliotheken vorhanden ist. Der gut funktionierende Buchhandel mit Lieferrouten von Nürnberg und Frankfurt am Main bzw. Mainz über Leipzig in das östliche Europa versorgte auch Breslau und Schlesien mit den Drucken aus dem deutschsprachigen und europäischen Raum. Nur angedeutet werden kann die Rolle der Buchmesse in Leipzig. Diese und andere kleinere Bibliotheken bildeten schließlich die Stadtbibliothek Breslaus, die als eine der bedeutendsten im östlichen Europa wie im deutschsprachigen Raum gelten kann.

Von den Adelsbibliotheken sei wenigstens auf die Reichsgräflich von Hochbergsche Majoratsbibliothek auf Schloss Fürstenstein hingewiesen (1067-1077). Sie wurde erst 1609 angelegt und umfasste zu diesem Zeitpunkt nur etwa 190 Werke, wuchs jedoch durch die verschiedenen Interessen ihrer Besitzer sehr schnell. So brachte z.B. Heinrich Ludewig Carl Graf von Hochberg 1738 von seiner Kavalierstour durch Europa „theils eine beträchtliche Sammlung rarer Bücher, theils allerhand 
Münzen und künstliche Instrumente“ mit (z.B. ein Newtonsches Teleskop) (1069). Einer der letzten Bibliothekare dieser Bibliothek, Karl Endemann, bezifferte ihren Umfang im Jahr 1907 mit 44.324 Bänden. Aber schon ihre Charakterisierung durch Gottfried Balthasar Scharff 1739 zeigt ihren geistesgeschichtlichen Stellenwert auf. Scharff war nicht nur protestantischer Pfarrer an der berühmten Friedenskirche zu Schweidnitz, sondern hinterließ auch ein umfangreiches, vor allem theologisches Werk, aber auch Dichtungen und gelehrtenAbhandlungen. Nicht zuletzt war er lange Zeit Herausgeber der Gelehrten Neuigkeiten Schlesiens, eines dieser Periodika, durch die zahlreiche Informationen aus der gelehrten Welt in Schlesien verbreitet wurden [Авв. 3].

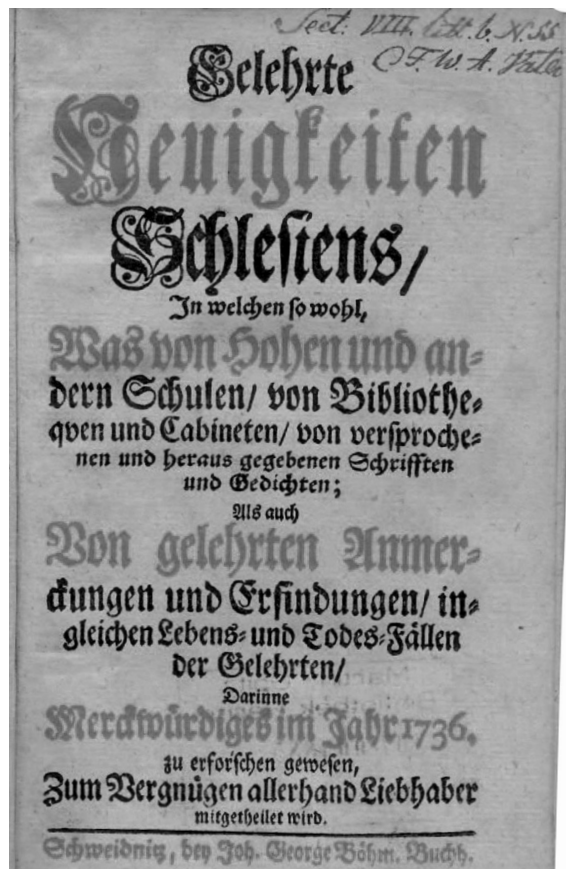

Abb 3. Titelblatt der Zeitschrift Gelehrte Neuigkeiten Schlesiens von

Pfarrer Gottfried Balthasar Scharff aus Schweidnitz.

Stiftung Martin Opitz Bibliothek, Herne, Sign. MOB RF 11. 
Es wäre hier noch einzugehen auf die Bibliotheken der Adligen Karl von Zierotin (883), des Herzogs Johann Christian von Brieg (1004), des Melchior von Wallenberg in Landeshut (1266), die der Grafen von Nostitz ursprünglich in Jauer (1330), die der Grafen von Oppersdorf in Oberglogau (1406) oder die der Fürsten von Oels (1425) oder auf die der Grafen von Schaffgotsch in Warmbrunn (1596) [Авв. 4].

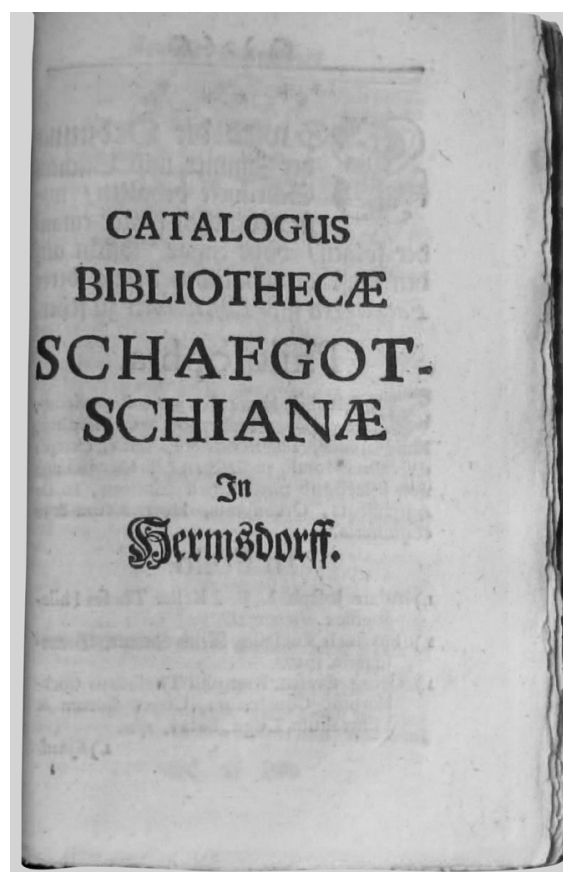

Abb 4. Titelblatt des Kataloges der Bibliothek der gräflichen Familie Schaffgotsch in Hermsdorf. Verfasser dieses Kataloges ist Pfarrer Gottfried Balthasar Scharff aus Schweidnitz. Er erschien 1738 in seiner Zeitschrift Gelehrte Neuigkeiten Schlesiens. Biblioteka Uniwersytecka w Wrocławiu, Sign. BUWr 375080/V. 
Zur Nostitzschen Bibliothek mit ihren immerhin 4.651 Bänden ist wenigstens zu bemerken, dass sie Anfang des 19. Jahrhunderts von der Knihovna Josefa Dobrovského in Prag erworben wurde (1334). Sie ist jetzt durch ein Repertorium vollständig erschlossen..$^{12}$ (1336 + MOB).

Als letztes sollen die Gelehrtenbibliotheken betrachtet werden. Sie sind eigentlich ein Thema für einen eigenen Beitrag. Aus der einleitenden historischen Skizze ist ersichtlich geworden, dass sich Gelehrte - d.h. Schulprofessoren, Theologen, Philologen, Mediziner, Naturhistoriker oder Historiker Schlesiens - ein eigenes Instrumentarium für ihre Studien schaffen mussten, da es keine Universität in Schlesien gab. Und, anders als heute, war der Weg von einer der kleineren Städte oder gar Dörfer nach Breslau mindestens beschwerlich, und man überlegte es sich sehr, ob man die Kosten für die Fahrt und Übernachtung wirklich auf sich nehmen sollte. Allerdings waren ihre Netzwerke über ganz Europa gespannt, so dass sie ihre Bibliotheken und regulären Kauf, aber auch durch Tausch und Geschenk erweitern konnten.

Die Reihe der Bibliotheken von Gelehrten wird mit dem Humanisten und Bischof Johannes IV. Roth (1426-1506) angeführt, der außer einer Reihe Theologica auch medizinische Handschriften, Botanica und Geographica besaß (852). Zu den Humanistenbibliotheken zählen die von Heinrich Rybisch (1485-1544) (853-855), die des genannten Thomas Rehdiger und seines älteren Bruders Nikolaus (1525-1616) (857), sowie die von Andreas Dudith (1533-1589), die wohl ursprünglich um die 5-6.000 Bände umfasste (ca. 3.700 Titel), von denen heute leider nur 340 nach den Forschungen von József Jankovics und István Monok (858) nachweisbar sind. Erwähnt werden müssen Johannes Crato von Krafftheim (1519-1585), Humanist und kaiserlicher Arzt, dessen Sammlung schon Anfang des 18. Jahrhunderts Aufmerksamkeit erregte (856). Bemerkenswert ist auch die Bibliothek der gelehrten Bibliophilen der Familie Hanke. Das Leben von Johannes, Martin und Gottfried umspannte die Zeit vom späten 16. bis zum frühen 18. Jahrhundert, Frankfurt a. M. [u.a.] 2015 (mit CD-Rom). 
ihre (bislang nicht erforschte) Bibliothek umfasste 4.141 Titel, deren Hauptbestandteile Theologie, Geschichte, Rechtswissenschaft und Philosophie und Philologie sind (894-896). An diesem Beispiel wird deutlich, dass Bibliotheksgeschichte auch Gelehrtengeschichte ist, dass sich anhand der Bestände und der Viten und Schriften ihrer Besitzer der Grad der Gelehrsamkeit und die regionalen, nationalen und internationalen Netzwerke erschließen lassen.

Es ist bemerkenswert, dass eine Bibliothek wie die der Hankes keinen Ausnahmefall für den Umfang von Privatbibliotheken in Schlesien darstellt. Solche mit mehr als 4.000 Bänden sind keine Seltenheit: Hier sind etwa der Breslauer Stadtkämmerer Zacharias Rampusch von Rammenstein (1632-1697) (908), der Rektor des Maria-MagdalenaGymnasiums und Kulturhistoriker Schlesiens Christian Stieff (16751751) (922-923) und der Theologe und Gesangbuchautor Johann Friedrich Burg (1689-1766) (929-930) zu nennen. Christian Gryphius (1649-1706), der Sohn von Andreas Gryphius, besaß 4.634 Bücher (911914), zwei Drittel davon lateinische und deutsche Ausgabe, ein Drittel Werke in anderen Sprachen.

Mehr als 6.000 Bücher umfassten etwa die Sammlungen des Pastors an der Heilig-Geist-Kirche in Breslau Gottlieb Jachmann (1686-1756) (928) und des Buchhistorikers Johann Ephraim Scheibel (1736-1809) (948-949).

Auf mehr als 9.000 Bücher hat es Johann Kaspar Arletius (1707-1784) gebracht, Historiker und Bibliophiler aus Breslau (934). Aber der bereits erwähnte Scharff in Schweidnitz - Pastor und Herausgeber der Gelehrten Neuigkeiten Schlesiens - besaß ca. 10.000 Werke (1509); der Katalog der Versteigerung fand sich in der Schedrin-Bibliothek in St. Petersburg.

Die Bibliothek des Pfarrers Leopold Johann Scherschniks (1747-1814) in Teschen in Mährisch-Schlesien (1559-1567) hatte eine ähnliche Bedeutung und Größe wie die Somogyi-Bibliothek in Szeged für Ungarn. Selbst ein Pastor wie Johann Friedrich Fülleborn $(\dagger 1770)$, der in dem kleinen Ort Domanze bei Schweidnitz lebte und für die Kirchen- und Kulturgeschichte Breslau von Bedeutung ist, besaß 2.368 Werke, vor 
allem natürlich Theologica, aber auch 630 geographische Karten, die von einem außerordentlichen nicht-theologischen Interesse sprechen (1036). Erwähnenswert ist auch die Bibliothek des aus einer schottischen Familie stammenden, aber in Polen naturalisierten John Johnstone (1603-1675), der als Naturforscher und Arzt im 17. Jahrhundert in Ziebendorf bei Lüben in Niederschlesien wirkte. Seine vorwiegend naturwissenschaftlich ausgerichtete Bibliothek mit ca. 4.000 Bänden möchte vielleicht nicht herausragen, aber er besaß überdies zahlreiche fremdsprachliche Werke, so dass der gesamte europäische Bildungskanon seiner Zeit vorhanden war (1622-1627).

Etwas später als Johnstone lebte Johann Gottlieb Milich (1678-1726), dessen Bibliothek sich heute in der Oberlausitzischen Bibliothek der Wissenschaften in Görlitz befindet. Sie ist durch eine Reihe von Katalogen erschlossen. Mit 4.000 Büchern, 200 Handschriften, 500 Münzen und zahlreichen Kuriositäten wurde sie 1726 Eigentum der Stadt Görlitz und der Grundbestand der oben genannten Einrichtung. Sie hat aufgrund ihrer inhaltlichen Vielfalt und Qualität schon früh die Aufmerksamkeit der Buchhistoriker gefunden (1135-1145).

Aus dieser kleinen Skizze mag ersichtlich geworden sein, dass die Bibliothekslandschaft Schlesiens mit ihren verschiedenen Bibliothekstypen (die es in anderen Landesteilen natürlich auch gab) in einem Maße bereichert wurde, wie man es sich heute kaum vorzustellen vermag. In Ermangelung tiefergehender Analysen sind hier vor allem die quantitativen Daten hervorgehoben worden, die allerdings bereits eine erhebliche Aussagekraft haben: Es zeigt sich, dass in Schlesien bis zur Gründung der Landesuniversität in Breslau 1702 eine beachtenswerte Bildungsbreite zu finden ist. Zahlreiche Theologen, Naturwissenschaftler, Historiker und an der Landeskunde Schlesiens interessierte Lehrer haben Bibliotheken aufgebaut, die ihre Spezialinteressen dokumentieren. Dadurch wurde eine immense Menge an überregionaler Literatur in Schlesien konzentriert. Leider sind viele dieser Bibliotheken nicht mehr physisch erhalten, aber Versteigerungskataloge legen von ihren 
Inhalten Zeugnis ab. ${ }^{13}$ In der Universitätsbibliothek in Breslau sind jedoch zahlreiche Versteigerungs- und Verkaufskataloge vorhanden, mit deren Hilfe man nicht nur die Umfänge, sondern auch die Bestände an Inkunabeln, Frühdrucken etc. bis ins Einzelne nachvollziehen kann. Das mag vielleicht etwas dürr erscheinen, aber es ist ersichtlich geworden, dass die Bibliothekslandschaft Schlesiens keineswegs nur ein regionales Phänomen war.

Auch die institutionell gesammelten Bücherschätze haben die Jahrhunderte nicht unbeschadet überdauert: Immer wieder haben, wie in anderen Bibliotheken des In- und Auslandes auch, Dublettenverkäufe stattgefunden und wurden dadurch Sammlungszusammenhänge auseinandergerissen, die kaum mehr vollständig rekonstruiert werden können. Schließlich ist, wie oben schon angedeutet, die Säkularisation ein weiterer Vorgang gewesen, der die Bestände von Klöstern und anderen kirchlichen Einrichtungen vielfach verstreut hat. Man war zu Beginn des 19. Jahrhunderts nicht der Auffassung, dass die Kenntnis der Genese einer Bibliothek für die Geistes- und Bildungsgeschichte von Nutzen sein könne. Das wird heute anders gesehen. Und schließlich haben die schwedische Besatzung im 17. Jahrhundert und der Zweite Weltkrieg und seine Folgen weitere Lücken in die Bestände gerissen.

Schon früh zog die vor allem in Breslau beheimatete Gelehrsamkeit und Bibliophilie weitere gelehrte Sammler an, und diese brachten das europäische Wissen in Form von Büchern mit. Schlesien ist also nicht nur eine „Brückenlandschaft", wie es gern in der Geschichtsund Kulturwissenschaft im Grunde implizit einschränkend genannt wird, ohne dass man das beabsichtigte, sondern hier konzentrierte sich auf hohem Niveau das Wissen in allen Disziplinen. Unterstützt wurde dies natürlich, worauf hier nur verwiesen werden kann, durch ungezählte Kontakte der schlesischen Gelehrten mit ihren europäischen Gesprächspartnern.

Es muss an dieser Stelle noch eine kurze Bemerkung zur Forschungsgeschichte eingefügt werden. Obwohl der Reichtum der 
schlesischen Bildungs- und damit Bibliotheks- und Buchgeschichte immens ist, ist leider festzustellen, dass die Forschungsintensität dramatisch gesunken ist. Dies konnte bei der Erarbeitung der oben genannten kommentierten Bibliographie festgestellt werden. Waren es bis 1945 vor allem deutsche Buchhistoriker, die sich den schlesischen Bücherschätzen und der Druckgeschichte in Schlesien widmeten, so ist die Erschließung dieser Schätze die Domäne polnischer und tschechischer Forscher. In den 1950er bis 1970er Jahre haben Gelehrte wie Marta Burbianka, Alodia Kawecka-Gryczowa, Józef Mandziuk, Aleksandra Mendykowa, Krzysztof Migoń, Aleksander Rombowski oder Adam Skura unser Wissen über die schlesische Buch- und Bibliotheksgeschichte erheblich erweitert. Naturgemäß war in der DDR und in der Bundesrepublik Deutschland das Interesse an den im „fernen“ Polen unerreichbaren Bibliotheken sehr gering. Nach 2010 ist keine signifikante Publikationstätigkeit mehr festzustellen. Obwohl nun seit fast 30 Jahren die Grenzen geöffnet sind, hält sich auch das Interesse deutscher Historiker deutlich in Grenzen und auch das der polnischen und tschechischen Wissenschaftler geht klar wahrnehmbar zurück.

Immerhin - und damit soll dieser kleine Beitrag abgeschlossen werden - diese Schätze sind in Breslau, in Prag und an anderen Orten wohlverwahrt und es bedarf keiner großen Anstrengungen mehr, sie in die Hand zu nehmen. In Breslau vergehen zwischen Bestellung eines Buches und dessen Lieferung auf den Arbeitstisch maximal rund 15 Minuten. Und das ist doch eine positive Perspektive! 
LES BIBLIOTHÈQUES ET L'ÉCONOMIE DES CONNAISSANCES Bibliotheken und die ÖKonomie des Wissens

$$
145^{\circ}-185^{\circ}
$$

Colloque international - Internationale Tagung 9-13 avril/April 2019 Sárospatak (Hongrie/Ungarn)

Édité par

Frédéric Barbier, István Monok et Andrea Seidler 


\section{L'Europe en réseaux}

Contribution à l'histoire de la culture écrite 1650-1918

Vernetztes Europa

Beiträge zur Kulturgeschichte des Buchwesens 1650-1918

Édité par

Frédéric Barbier, Marie-Elisabeth Ducreux, Matthias Middell, István Monok, Éva Ringh, Martin Svatoš

Volume VIII

École pratique des hautes études, Paris

École des hautes études en sciences sociales, Paris

Centre des hautes études, Leipzig

Bibliothèque nationale Széchényi, Budapest

Bibliothèque et centre d'information de l'Académie hongroise des sciences, Budapest 
LeS BIBLIOTHÈQUeS ET L'ÉCONOMIE DES CONNAISSANCES BibliotheKen und die ÖKonomie des Wissens

$$
145^{\circ}-185^{\circ}
$$

Colloque international - Internationale Tagung 9-13 avril/April 2019 Sárospatak (Hongrie/Ungarn)

Édité par

Frédéric Barbier, István Monok et Andrea Seidler

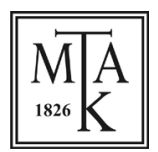

Magyar Tudományos Akadémia Könyvtár és Információs Központ Budapest 2020 
Mise en page

Viktória Vas

ISBN 978-963-7451-57-7

DOI 10.36820/SAROSPATAK.2020 
Préface

István MoNOK

Bibliothecae mutantur - Quare, quemadmodum et quid attinet?

Transformations de la composition thématique

des bibliothèques du Royaume de Hongrie aux $\mathrm{XV}^{\mathrm{E}}-\mathrm{XVI} \mathrm{I}^{\mathrm{e}}$ siècles....11

Marianne CARbonnier-Burkard

Les bibliothèques des Églises réformées françaises au XVII siècle.... 30

Max Engammare

De la bibliothèque de l'Académie de Calvin (1570) a la bibliothèque de l'Académie de Bèze (1612) à travers leur catalogue: Continuités et ruptures jusqu'au troisième catalogue de 1620........... 57

Róbert OLÁ́

Obsolescent Reformed Libraries in the seventeenth and eighteenth Century Carpathian Basin

Ádám Hegri

Moderner Zeitgeist - veraltete Lesestoffe. Bibliotheken reformierten Pfarrer um die Wende des 18. zum

19. Jahrhunderts im Königreich Ungarn

Petr MAšEK

Zierotin Library in Velké Losiny in Sixteenth and Seventeenth century. 136

Detlef HABERLAND

Schlesische Bibliotheken Zeichen der intellektuellen Vielfalt einer zentralen Bildungsregion in Europa 146

Thomas WaLLNIG

Sebastian Tengnagel und Johann Seyfried - Österreichische

Geschichtsschreibung zwischen Späthumanismus und

Gegenreformation 
Elisabeth EngL-Ursula RautenBerg

Christoph Jacob Trew - Bibliothek und Sammeln in der Gelehrtengemeinschaft der ersten Hälfte des 18. Jahrhunderts.

Helwi BLOM

Philosophie ou Commerce?

L'évolution des systèmes de classement bibliographique dans les catalogues de bibliothèques privées publies en France au XVIII ${ }^{\mathrm{e}}$ siècle.

Maria Luisa López-VIDrIero Abelló

Les meubles de la connaissance: façons de devenir sage à prix fixe.

Frédéric BARbier

Distinction, récréation, identité: la trajectoire des « romans» en France sous d'Ancien Régime.

\section{Andrea SeIdler}

Die praktische Bedeutung ungarischer Sammlungen und Bibliotheken für führende Gelehrte des Königreichs Ungarn im späten 18. Jahrhundert am Beispiel des Jesuiten Georg Pray (1723-1801).

\section{Olga Granasztói}

Se divertir: les enseignements de la bibliothèque d'une femme aristocrate hongroise à la fin du XVIII siècle

Christophe Didier

La naissance du théâtre „des boulevards”, ou Comment la banlieue entre en bibliothèque (1780-1830).

\section{Andrea De Pasquale}

La nascita delle riserve di libri antichi in Italia

Index des noms de personne et de lieu..... 